\title{
Stage IIIC Prostate Cancer AJCC v8
}

National Cancer Institute

\section{Source}

National Cancer Institute. Stage IIIC Prostate Cancer A/CC v8. NCI Thesaurus. Code C140172.

Stage IIIC includes: Any T, N0, M0, PSA: Any, Grade Group 5. N0: Prostate cancer with no positive regional nodes. M0: Prostate cancer without evidence of distant metastasis. Grade Group 5: Gleason Score 9 or 10, Gleason Pattern 4+5, or 5+4, or 5+5. (AJCC 8th ed.) 